\title{
Procesos Destructores para la Salud Vinculados a la Manipulación de Agroquímicos en Trabajadores Agrícolas de Young, Uruguay
}

\section{HEALTH DESTRUCTIVE PROCESSES RELATED TO THE HANDLING OF AGROCHEMICALS IN AGRICULTURAL WORKERS IN YOUNG, URUGUAY}

\author{
Jimena Heinzen', Nicolás Rodríguez² \\ 1. Ex Asistente del Polo de Salud Comunitaria. Sede Paysandú del Centro Universitario Regional Litoral Norte, Universidad de la República. Paysandú, Uruguay. \\ 2. Asistente del Polo de Salud Comunitaria. Sede Paysandú del Centro Universitario Regional Litoral Norte, Universidad de la República. Paysandú, Uruguay.
}

\begin{abstract}
RESUMEN
Uruguay no ha estado ajeno a los cambios acontecidos en lo que hace al cultivo de granos en América Latina a partir del aumento del precio de los commodities a nivel internacional. Estos implicaron la expansión de la frontera agrícola conjuntamente con la adopción de siembra directa, la utilización de semillas transgénicas y la aplicación intensiva de agroquímicos. Esta modernización del espacio agrario no incorporó el acceso a la información para las poblaciones sobre los potenciales riesgos para su salud. Los trabajadores rurales que manipulan agroquímicos fueron identificados como una de las poblaciones con particular vulnerabilidad. Se llevó adelante un estudio exploratorio basado en la propuesta del Modelo Obrero Italiano como enfoque para el estudio de la salud de los trabajadores. Se realizaron 5 entrevistas a informantes clave a partir de una muestra de casos típicos, las que permitieron reconstruir los procesos de trabajo. Posteriormente se validaron estos resultados y se identificaron procesos protectores y destructores para la salud en talleres de discusión colectiva. De los resultados se destaca que los perfiles de toxicidad colectivos son producto de un modelo productivo determinado, donde la imposición del uso intensivo de agroquímicos es uno de los aspectos.
\end{abstract}

(Heinzen J, Rodríguez N, 2016. Procesos Destructores para la Salud Vinculados a la Manipulación de Agroquímicos en Trabajadores Agrícolas de Young, Uruguay. Cienc Trab. May-Ago; 18 [56]: 117-123).

Palabras clave: TRABAJADORES RURALES, RIESGOS LABORALES, AGROQUÍMICOS.

\section{ABSTRACT}

Uruguay has not been an exception on the exponential growth of grains crops that occurred in Latin America due to the rise or commodity prices internationally. These involved the expansion of the agricultural frontier, the adoption of direct seeding, the use of transgenic seeds and the intensive application of agrochemicals. This modernization of the agricultural space did not incorporate access to information for people about potential health risks. Agricultural workers that handle agrochemicals were identified as a specific vulnerable population. An exploratory study based on the proposal of the Italian Working Model as an approach to the study of the health of workers was carried out. For reconstruction of work processes were performed 5 interviews to key informant based on a sample of typical cases. Besides interviews, results were validated in groups; subsequently, protective and destructive processes to health were identified in collective workshops discussion. From the results, it stands that collective toxicity profiles occur in context of a specific production model, where the imposition of intensive use of agrochemicals is one of their aspects.

Keywords: RURAL WORKERS, OCCUPATIONAL HAZARDS, AGROCHEMICALS.

\section{INTRODUCCIÓN}

Uruguay ha experimentado en los últimos 15 años cambios en su sistema agropecuario, donde la introducción de soja resistente a herbicidas y la siembra directa extensiva tuvo un crecimiento exponencial. Este cultivo pasó de abarcar 10.000 ha en la zafra 2002/03 a cerca de 1,2 millones ha en la zafra 2012/13, desarrollándose de forma particular en la zona litoral oeste del país. ${ }^{1} \mathrm{La}$

\section{Correspondencia / Correspondence:}

Nicolás Rodríguez

Florida 1065. Paysandú, Uruguay. CP: 60000

Tel.: (00598) 47220221 • Fax: int. 104

e-mail: nicolasr@psico.edu.uy

Recibido: 29 de Febrero de 2016 / Aceptado 06 de abril de 2016 ciudad de Young se ha constituido en uno de los principales enclaves de producción agrícola del litoral, tanto por su accesibilidad geográfica como por la fertilidad de sus suelos. Esta ciudad se encuentra ubicada en el centro geográfico del departamento de Río Negro y cuenta con una población de 16.756 personas. ${ }^{2}$ El uso del suelo ha sido históricamente destinado a la producción ganadera, pero en los últimos años la frontera agrícola se ha expandido notablemente. En la actualidad se ha convertido en una de las regiones de mayor producción de cultivos de secano como cereales y oleaginosas: trigo, cebada, maiz, sorgo, girasol y soja. Paulatinamente, Young se convirtió en un emplazamiento estratégico de este tipo de cultivos, no sólo por las tierras fértiles de su área de influencia, sino también por su capacidad logística: compra de insumos y maquinaria, almacenamiento y transporte, servicios bancarios y administrativos. $^{3}$ Por esta razón, en todo el entorno geográfico de esta ciudad se instrumentó lo que se ha llamado paquete tecnológico de distintas empresas agrocapitalistas, el que se ha caracterizado por la adopción de la siembra directa, la utilización de semi- 
llas transgénicas y la aplicación intensiva de agroquímicos. ${ }^{4} \mathrm{La}$ expansión del agronegocio en el territorio reconfiguró las relaciones sociales de producción, donde la incorporación de nuevas tecnologías no fue acompañada por procesos de información a la población sobre los potenciales riesgos para su salud.

Esta incertidumbre sobre los impactos a la salud, principalmente los derivados de la utilización de agroquímicos, es lo que motiva que en el 2012 un colectivo de profesionales y pobladores de Young le solicite a la Universidad de la República la realización de algún tipo de estudio. A partir de este pedido, el Centro Universitario de Paysandú trabaja en una investigación participativa orientada a conocer las distintas dimensiones del problema. En este estudio exploratorio denominado "Saberes y prácticas en relación al impacto de los agroquímicos en la ciudad de Young", que involucró a trabajadores rurales, estudiantes y a la comunidad en general, se logró una aproximación al tema a partir de la cual se elaboraron ciertas conclusiones preliminares: a) la precarización laboral y la insuficiente capacitación para el manejo de agroquímicos coloca a los trabajadores rurales como una de las principales poblaciones de riesgo; b) a pesar de que la reglamentación en Uruguay establece distintas limitaciones para el manejo de agroquímicos, existen diferentes problemáticas en la aplicación de la normativa y en su control; y c) la población en general desconoce los riesgos para la salud y el ambiente emergentes de la tecnificación de la producción y de las sustancias que se utilizan.

A partir de esta primera aproximación al problema es que se decide ampliar el equipo disciplinariamente y formular un proyecto de investigación dirigido a monitorear participativamente la utilización de agroquímicos. Esta investigación fue denominada "Salud laboral y ambiental en torno a la utilización de agroquímicos en la localidad de Young. Estrategias de monitoreo participativo", y se desarrolló entre el 2013 y 2015 por parte de un equipo multidisciplinario, conformado por docentes de agronomía, geografía, medicina, psicología y química. A partir de la conformación de este equipo se pretendió generar un abordaje integral de la relación entre agroquímicos y salud colectiva, donde uno de los ejes estuvo dado por la exposición de los trabajadores rurales a estos productos con distintos niveles de toxicidad. Esta población había sido identificada como particularmente vulnerable y no existian estudios nacionales al respecto. En este artículo se presentan los resultados correspondientes a esta dimensión de la investigación, más específicamente, se describen y analizan los procesos destructores para la salud de los trabajadores expuestos a agroquímicos en cultivos de secano.

Los estudios tradicionales sobre salud y trabajo han resultado insuficientes para abordar esta problemática, ya que suelen centrarse en el trabajador aislado en su puesto laboral, y no reconocen la complejidad y multiplicidad de elementos que se ponen en juego en un proceso productivo. $\mathrm{Al}$ individualizar los riesgos se desconocen los impactos a la salud en el colectivo de trabajadores que desempeñan similares tareas, además de omitir el saber obrero acumulado a partir de la propia experiencia. ${ }^{5}$ Conocer el proceso de trabajo y las características de cada uno de los elementos que lo componen es indispensable para poder comprender los procesos peligrosos o saludables que se generan, así como para sugerir modificaciones y promover salud en el ámbito laboral. La forma en la que el objeto, los medios, la actividad y la organización del trabajo interactuarán en un determinado proceso laboral, produce distintos mediadores del proceso de salud-enfermedad. ${ }^{6}$ Esos mediadores son protectores cuando se orientan hacia la promoción de potencialidades y la creatividad de los trabajadores, o se configuran como destructores cuando adquieren formas de organización o utilizan medios que favorecen el desarrollo de determinados padecimientos. Los procesos de trabajo no favorecen exclusivamente procesos dañinos ni son enteramente beneficiosos, sino que se encuentran en una relación dialéctica, de modo que el trabajo adquiere condiciones contradictorias. ${ }^{7}$

En lo que respecta a la manipulación de agroquímicos, los estudios desde esta perspectiva son recientes en el tiempo y acompasan la profundización del capitalismo agrario que acontece a partir del siglo XXI. Al respecto, se destacan las investigaciones realizadas en el cono sur con trabajadores de la horticultura ${ }^{8}$, de la floricultura, ${ }^{9,10}$ y de monocultivos extensivos de Baixo Jaguaribe/CEBrasil. ${ }^{11}$ De esta manera, en base al marco teórico referencial sobre salud en el trabajo antes descrito y a través de un proceso de co-construcción de conocimientos, se desarrolló este estudio sobre las condiciones de trabajo de asalariados que manipulan agroquímicos en los cultivos de secano de la ciudad de Young. En este artículo se presentan las principales características del proceso de trabajo, se hace un énfasis particular en los modos de organizar el trabajo, y a partir de esto se describen y analizan los procesos destructores y protectores para la salud identificados por los trabajadores.

\section{MATERIAL Y MÉTODOS}

La investigación realizada se enmarca dentro de lo que Souza Minayo $^{12}$ denomina como investigación social en salud, en tanto se abordaron cuestiones vinculadas a los procesos de salud-enfermedad-cuidado a través de la mirada de los distintos actores y grupos sociales involucrados. El estudio realizado fue de características cualitativas, ya que es un abordaje que permite profundizar sobre los elementos que interactúan en un grupo delimitado o en relación a un proceso o fenómeno. ${ }^{12}$ El enfoque utilizado para cumplir el objetivo propuesto se basa en los aportes del Modelo Obrero Italiano (MOI) al estudio de la salud de los trabajadores. En este enfoque confluye el saber técnico en materia de salud y el saber obrero en materia de condiciones de trabajo bajo el precepto de no delegación de la salud. ${ }^{13}$ Originariamente la propuesta de investigación del MOI fue concebida para reconocer los riesgos para la salud en una empresa o fábrica y proponer soluciones particulares. En esta investigación y en estudios anteriores se ha utilizado en un sentido más general, ya que se tomaron rubros o cadenas productivas con trabajadores provenientes de distintas empresas. Esto posibilitó recuperar las particularidades de un sector y no la singularidad de un establecimiento productivo. ${ }^{14}$

En cuanto al diseño metodológico, la pregunta que orientó la investigación fue la siguiente: cómo es el proceso de trabajo en aquellos sectores de la producción de cultivos de secano que presentan mayor exposición a los agroquímicos, qué destructores y protectores para la salud identifican los trabajadores de la ciudad de Young y cuáles son los condicionantes de dichos procesos. Al no contar con una organización sindical que nucleara a los trabajadores de estos cultivos, se accedió a un listado de trabajadores que formaban parte de las bolsas de trabajo del Centro Público de Empleo (CEPE) dependiente del Ministerio de Trabajo y Seguridad Social. A través de la coordinación con el CEPE Young, se convocó a trabajadores agrícolas a la presentación del proyecto de investigación y a los participantes se los contactó para colaborar con el estudio. Asimismo, se utilizó la técnica 
de la bola de nieve ${ }^{15}$ para acceder a otros trabajadores, ya sea a partir de los que fueron convocados a través del CEPE y de otros vínculos en la ciudad. Esta técnica implicó que los trabajadores contactaran al equipo investigador con otros informantes, personas que cumplían los criterios de inclusión definidos y que estaban dispuestas a ser entrevistadas. $^{15}$

Se realizó una muestra de casos típicos ${ }^{16}$, donde los criterios de inclusión estuvieron dados por personas con experiencia actual o pasada de trabajo en cultivos de secano, y que hayan tenido contacto directo con agroquímicos en maquinarias de aplicación área o terrestre. En función de esta delimitación, los puestos de trabajo comprendidos fueron el de peón de campo o tractorista, mosquitero*, aguador y piloto de avión. La muestra quedó conformada así por trabajadores agrícolas que tenían una exposición cotidiana a los agroquímicos en las distintas etapas del cultivo. No se tomaron en cuenta otros puestos del trabajo del sector vinculados a la preparación de semillas, transporte y acopio de granos. Con esta decisión se pretendía abarcar un universo específico de trabajadores, pero no se desconoce que los asalariados de otros sectores pueden tener similares niveles de exposición a los seleccionados.

En lo que respecta al proceso de investigación, este se implementó en 3 etapas en función de los momentos del cultivo y de las posibilidades de los trabajadores para participar en actividades luego de la jornada laboral. La primera etapa tuvo como objetivo reconstruir el proceso laboral en los cultivos de secano de la zona de influencia de Young a través de entrevistas en profundidad a trabajadores que desempeñaban tareas en el sector. ${ }^{12}$ Se realizaron un total de 5 entrevistas a informantes clave, un trabajador de cada uno de los puestos identificados para la muestra, y a un docente de la Universidad de la República, Ingeniero Agrónomo, referente en sistemas de producción.

A partir del análisis de contenido de este material se elaboraron esquemas que recogían los momentos de cultivo y las tareas que se desempeñaban en cada una de las etapas. ${ }^{12}$

La segunda etapa estuvo dada por la validación colectiva del proceso laboral, y la identificación de los procesos protectores y destructores para la salud presentes en el proceso de trabajo. La técnica utilizada fue el dispositivo del taller, ya que se buscó facilitar la participación, la recuperación del saber obrero y la co-construcción de conocimiento entre el equipo universitario y los trabajadores. ${ }^{14,17}$ Se realizaron 2 talleres en los que participaron aproximadamente 15 personas. Los esquemas elaborados a partir de las entrevistas fueron adecuados a un formato de tabla para

\footnotetext{
* Denominación utilizada para el trabajador/a encargado de la operación del "mosquito", forma en que se denomina a un tipo específico de maquinaria agrícola que realiza fumigación terrestre.
}

facilitar su comprensión. Estas tablas oficiaron de disparadores de la discusión de los talleres; en una primera instancia se validaron los elementos aportados en las entrevistas, se incorporaron nuevas tareas, se señalaron diferencias entre distintos establecimientos, así como diferencias entre los trabajadores en el desempeño de las tareas. En un segundo momento se trabajó en la identificación de procesos protectores y destructores para la salud vinculados al uso de agroquímicos. Los investigadores actuaron como moderadores, a través de la pregunta y de la búsqueda de consensos para validar colectivamente los datos. Los talleres, así como las entrevistas, fueron registrados en audio digital y su contenido fue transcripto. La tercera etapa contempló la sistematización de lo emergente y la consiguiente devolución e intercambio con los trabajadores y la comunidad de Young. Se realizó una jornada de cierre de la investigación en la que se presentaron los principales resultados y se intercambió en torno a una propuesta de monitoreo participativo en salud ambiental y laboral. En dicha actividad participaron autoridades nacionales y locales de los organismos competentes, vecinos y vecinas preocupados por la problemática, técnicos, trabajadores $\mathrm{y}$ pequeños productores.

Durante el trabajo de campo participaron aproximadamente 20 trabajadores, el cual culminó cuando se logró relevar la información necesaria sobre los distintos puestos de trabajo ya mencionados. En lugar de utilizar el criterio de saturación teórica se optó por cubrir el espectro de tareas, en tanto fue un estudio de aproximación al campo de problemas. ${ }^{12,15}$ Los trabajadores participaron voluntariamente, en todos los casos se realizó el proceso de consentimiento informado, donde se explicaron las implicaciones del estudio y se garantizaba la confidencialidad de los datos. El proyecto de investigación fue evaluado y aprobado para su implementación por el Comité de Ética de la Facultad de Psicología de la Universidad de la República (Exp. № 191130-000695-12).

\section{RESULTADOS}

En el marco de la investigación más amplia ya descrita se elaboró una caracterización productiva del área de influencia de la ciudad de Young a través del relevamiento de datos secundarios. ${ }^{3}$ Dicho estudio permite concluir que los momentos de la producción, así como los procesos de trabajo, se dan de forma cíclica más allá del cultivo, distinguiéndose únicamente las épocas del año según sea producción de verano o invierno. En la Tabla 1 se presenta la reconstrucción del proceso laboral en términos generales. A cada tarea corresponde una descripción de las actividades que constituyen cada momento productivo.

Tabla 1.

Procesos de trabajo según las etapas en cultivos de secano de verano (Ej: soja).

\begin{tabular}{|c|c|c|c|c|c|c|}
\hline Meses & Momentos & Procesos de Trabajo & & & & \\
\hline Ago - Set & Preparación del suelo & Inoculado y curado de semillas & $\begin{array}{l}\text { Carga y descarga } \\
\text { de bolsas }\end{array}$ & $\begin{array}{l}\text { Manejo del tractor } \\
\text { para nivelado }\end{array}$ & $\begin{array}{l}\text { Preparación de } \\
\text { agroquímicos }\end{array}$ & $\begin{array}{l}\text { Operación del mosquito/ } \\
\text { tractor/avión } \\
\text { para aplicación }\end{array}$ \\
\hline Nov - Dic & Siembra de 1 a y $2 a$ & Operación de sembradora & Control de siembra & Abonado & $\begin{array}{l}\text { Preparación de } \\
\text { agroquímicos }\end{array}$ & $\begin{array}{l}\text { Operación del mosquito/ } \\
\text { tractor para aplicación }\end{array}$ \\
\hline Dic - Feb & $\begin{array}{l}\text { Control de malezas, } \\
\text { plagas y riego }\end{array}$ & Operación de riego & & & $\begin{array}{l}\text { Preparación de } \\
\text { agroquímicos }\end{array}$ & $\begin{array}{l}\text { Operación del mosquito/ } \\
\text { tractor/avión } \\
\text { para aplicación }\end{array}$ \\
\hline Mar - May & Cosecha & Operación de cosechadora & Manejo de tractor & $\begin{array}{l}\text { Operación de } \\
\text { carro granelero }\end{array}$ & $\begin{array}{l}\text { Preparación de } \\
\text { agroquímicos }\end{array}$ & $\begin{array}{l}\text { Operación del mosquito/ } \\
\text { tractor para aplicación }\end{array}$ \\
\hline
\end{tabular}

Fuente: elaboración propia. 
El análisis del proceso de trabajo permitió identificar los procesos peligrosos y protectores para la salud de los trabajadores. Asimismo, como elemento transversal se analizó la organización del trabajo en la manipulación de agroquímicos y su relación con dichos procesos. A continuación, se detallan los resultados de ambos aspectos.

\section{Organización del trabajo en la manipulación agroquímicos}

En lo que hace a la organización del trabajo en las entrevistas y talleres realizados se destacó que a los trabajadores se les solicita que cubran determinada área o tarea concreta, dejando la organización de la jornada a su criterio (no de todo el proceso). Esto es valorado positivamente por los trabajadores, en tanto no tiene la presencia de los capataces de forma constante sobre ellos y lo que hacen, pero a su vez evidencia la soledad en la que estos trabajadores transitan su jornada laboral. Asimismo, implica la responsabilización del trabajador sobre el predio, la tarea y los resultados; lo que contrasta con la escasa posibilidad de control o de aporte creativo al proceso de trabajo por parte de los asalariados. Las tareas que se realizan son caracterizadas como monótonas, dado que se repiten a lo largo de la jornada laboral y durante todo el ciclo del cultivo. A pesar de tratarse de un trabajo monótono, el mismo exige gran concentración, por lo que lo primero suele complotar para el logro efectivo de lo segundo.

Con respecto a la duración de la jornada laboral, se pudo observar que está fuertemente ligada a la forma de pago en el sector: por hora trabajada en el caso del peón, por hectárea en el caso de mosquiteros y por tarea para los pilotos. Los propios trabajadores plantean que deben realizar jornadas extensas para poder completar un mejor salario. La remuneración a destajo implica que no se contempla la nocturnidad ni las horas extras, más allá de las horas o del turno en el que se trabaje (en el caso de ciertos peones sí se cubrían estos derechos laborales). El ejemplo paradigmático son los mosquiteros y aguadores, estos trabajadores están a disposición de la empresa durante todo el año: "Te llaman, yo siempre estaba a la orden, en media hora el aguador te pasa a buscar" (Entrevista 2). Otros trabajadores plantean que tienen un sueldo fijo por día y un incentivo por hectárea realizada, lo cual fomenta la necesidad de cubrir la mayor cantidad de hectáreas posibles.

Durante el trabajo de campo de la investigación fue constante la mención a los ritmos acelerados de trabajo: "Es un trabajo de andar súper rápido, cuando se trabaja, se trabaja, hay mucho trabajo, mucha demanda de trabajo, y el empleado tiene el beneficio de la hectárea, entonces también aprovechamos" (Entrevista 2). En una tarea que se requiere concentración y cuidados a la hora de manipular productos tóxicos, la velocidad con la que se realiza la tarea obstaculiza la posibilidad de protegerse. Los trabajadores coinciden en señalar que si toman todas las precauciones, por ejemplo incorporar el uso de los equipos de protección personal (EPP), demorarian más de lo deseable $y$, consiguientemente, accederían a pagos inferiores.

\section{Procesos destructores para la salud}

En cuanto a los procesos destructores identificados, el contacto con agroquímicos es planteado como el principal proceso destructor. Si bien todos los trabajadores estaban en contacto con estas sustancias, como se pudo apreciar en la Tabla 1, el proceso de trabajo abarca otra serie de aspectos. Los demás procesos destructores aparecen como condicionantes, moduladores o potenciadores de los procesos vinculados al contacto con agroquímicos. Durante el proceso de trabajo se identificaron distintos momentos en que los trabajadores toman contacto con los agroquímicos: al cargar y transportar en el camión hasta el predio; al realizar fraccionamientos, diluciones y mezclas para la aplicación; al cargar el mosquito, el tractor o el avión; durante la aplicación en sí; y al limpiar los envases para descartar. En todos estos procesos el contacto puede darse directamente a través de la piel y mucosas en el caso de accidentes por salpicaduras; así como por vía inhalatoria al respirar mientras se mezcla o se aplican agroquímicos. La preparación de agroquímicos es la tarea principal del aguador, actividad que se repite numerosas veces a lo largo de la jornada laboral.

Es importante subrayar que la exposición no es eventual o fortuita, tampoco es exclusiva de los posibles accidentes que se produzcan, sino que se da de forma permanente. Otro elemento observado es que a menor calificación de los trabajadores el contacto con los agroquímicos es mayor. Peones, aguadores y apoyos de tierra son quienes se encargan del transporte, preparación y disposición final de los productos tóxicos utilizados en el cultivo, mientras que mosquiteros y pilotos están a cargo de controlar este proceso y operar la maquinaria. En entrevistas y talleres se constata un escaso uso de los EPP. Esto se debe a múltiples factores: escasa disponibilidad por parte de las empresas, falta de adaptación de los equipos a las tareas, confianza del trabajador o la no consideración de su uso. Esta carencia se configura como un proceso destructor en tanto aumenta el contacto de los trabajadores con los agroquímicos y en caso de accidentes los expone directamente.

Otros elementos que condicionan una mayor exposición de los trabajadores a los agroquímicos están vinculadas a la forma de organizar el trabajo: jornadas extensas, falta de descansos o descansos escasos, trabajo nocturno y monótono, así como los ritmos laborales acelerados. Estos fueron identificados como destructores en tanto aumentan la exposición en términos temporales y, simultáneamente, aumentan las posibilidades de sufrir accidentes durante la operación de la maquinaria, al manejar en ruta o en la preparación de los productos. El contacto con agroquímicos por vía inhalatoria se da también durante la aplicación, en el caso de los peones se da por la falta de cabina o de filtros adecuados cuando los tractores son cabinados. Además, están expuestos cuando se está aplicando con mosquito y ellos están realizando otro tipo de tareas en el predio, como es el caso de los aguadores. Si bien tienen precauciones en cuanto al viento y la deriva que se genera, estos trabajadores están expuestos en gran medida a los productos durante la aplicación. En el caso del piloto también se da exposición durante la aplicación dado que las cabinas de los aviones no suelen ser herméticas e ingresa parte de las sustancias aplicadas.

Por último, se destaca como proceso destructor la falta de decisión sobre el proceso laboral. A pesar de que los trabajadores relatan que tienen ciertos niveles de libertad para organizar su jornada, no tienen capacidad de decisión sobre la posibilidad de aplicar o no cuando las condiciones climatológicas parecerían indicar que no debería aplicarse, por citar un ejemplo. En el caso del piloto, se destaca como proceso destructor en la organización del trabajo la responsabilidad sobre las consecuencias negativas que puedan generarse durante la aplicación (ej.: fumigar un cultivo lindero o una escuela rural). Si bien esto está reglamentado y los pilotos conocen la reglamentación y toman distintas medidas de control y previsión para evitar errores; en caso de que ocurran, fue mencionado que los costos son compartidos con la empresa. Este proceso de trabajo, si bien fue valorado como protector en tanto permite tomar decisiones y controlar en cierta medida el proceso laboral, 
tienen su contrapartida destructora que puede expresarse en impactos psicosociales para los trabajadores. En esta misma línea se mencionaron las presiones de parte de productores y de las empresas para que se realicen las aplicaciones a pesar de que no están dadas las condiciones.

\section{Procesos protectores para la salud}

En cuanto a los procesos protectores, peones y tractoristas señalaron la disponibilidad de EPP como único protector en sus procesos de trabajo. Mosquiteros y aguadores destacaron el trabajo en equipo; si bien esto varía según las relaciones personales entre mosquitero y aguador, la posibilidad de estar acompañado durante la jornada laboral es valorada positivamente. Otro proceso protector señalado en el caso del aguador es la posibilidad de tiempos de descanso mientras el mosquitero aplica. Aunque tienen tareas para desempeñar entre carga y carga del mosquito, los participantes señalaron que disponen de tiempo para descansar. Por último, se plantea como proceso protector la disponibilidad de EPP cuando éstos están presentes y son de buena calidad: "Claro, el equipo adecuado, o sea, muchas veces no son... Hay equipos que le pasa el agua, o sea, esos equipos ya no...” (Taller 1). En el caso de los pilotos de avión, los trabajadores entrevistados identificaron la disponibilidad de EPP tanto para el apoyo de tierra como para el piloto. Además, se destacó la disponibilidad de agua y jabón para el lavado de manos luego de la preparación de agroquímicos. Otro proceso protector señalado fue la posibilidad de conocer los productos que se aplican, recibirlos en buenas condiciones y con la garantía de las boletas de agrónomos. A su vez, la ya mencionada capacidad del piloto de controlar si se aplica o no en función de la evaluación realizada también se señaló como un aspecto benéfico para su autocuidado.

\section{DISCUSIÓN}

Ya sea por el objetivo del proyecto como por lo consignado en los talleres, el contacto con agroquímicos fue identificado por los trabajadores como el principal proceso destructor. Dicho contacto se da en distintos momentos del proceso de producción y a través de las diversas tareas que se desarrollan. Interesa señalar que la presencia de determinados objetos y medios en el proceso laboral no son azarosos, ni son la única forma posible de desarrollar la agricultura. Los trabajadores están en contacto con los agroquímicos, dado que los mismos son su objeto de trabajo; de ahí que más allá de las precauciones tomadas por los trabajadores o de los equipos de protección brindados, el proceso peligroso surge del modelo productivo y sus procesos de trabajo. En este contexto, el análisis de los procesos destructores y sus posibles impactos en la salud de los trabajadores no puede realizarse desde la noción de riesgo de la epidemiología tradicional. La exposición de los trabajadores a los productos químicos utilizados en la producción no es contingente o más o menos probable según factores aislados y externos, sino que forma parte de un determinado modelo de producción. La retórica de la justificación ha sido parte de la lógica argumentativa de quienes promueven el uso intensivo de estas sustancias. ${ }^{18}$

$\mathrm{Al}$ respecto, Breilh ${ }^{7}$ plantea que si se desconoce el carácter permanente de determinados procesos destructores se los está desvinculando de la totalidad que los produce y fundamenta. Los trabajadores que se desempeñan en cultivos de secano de la zona de influencia de Young están expuestos de forma crónica a distintos agroquímicos utilizados para la producción. Dicha exposición implica un patrón que se repite porque está enmarcado en un modo de vida colectivo, se presenta de forma cotidiana y repetida a lo largo de la jornada laboral y en todo el proceso de cultivo. Esta imposición se define como exposición permanente o inherente dado que no varía y el sujeto no puede librarse de ella. ${ }^{7}$ Es así que no se puede analizar la exposición a los agroquímicos descontextualizada del conjunto de elementos que componen el proceso de trabajo. La confluencia de procesos macro y microestructurales imprimen modalidades de trabajo particulares y pautan perfiles de riesgo colectivos entre los trabajadores. Durante la investigación esto pudo ser constatado en tanto trabajadores de distintas empresas y establecimientos compartían los elementos estructurantes del trabajo en los cultivos de secano y validaron los distintos procesos peligrosos y protectores en un sentido general en el sector.

Además de estar expuestos de forma crónica a los agroquímicos, a los trabajadores se les imponen distintos elementos vinculados fundamentalmente a la forma de organizar y dividir socialmente el trabajo. Entre ellas se destacaron las formas de contratación precaria, el pago a destajo, las extensas jornadas de trabajo con breves o nulos tiempos de descanso, los ritmos acelerados para realizar las tareas, así como la falta de poder de decisión o aporte creativo sobre las tareas. A los efectos de este estudio, se focalizó en la manipulación de agroquímicos, pero su connotación como proceso peligroso sólo adquiere sentido si se vincula con los aspectos mencionados en cuanto a la organización del trabajo. La precariedad laboral observada en Young no es ajena a lo que acontece con otros trabajadores rurales del país. Los contratos a término, los ingresos económicos insuficientes y una inadecuada seguridad social, pautan condiciones objetivas y subjetivas de trabajo tendientes a expropiar al asalariado las posibilidades de control de su fuente de empleo y sus condiciones de vida en general. ${ }^{19}$ En la afirmación precedente se puede agregar a los procesos de salud-enfermedad-cuidado, ya que tanto en esta investigación como en estudios anteriores ${ }^{20}$ se pudo observar que cuanto más precario es el trabajo, los procesos peligrosos son más acentuados.

Por otro lado, en lo que hace a la exposición permanente a los agroquímicos, los equipos de protección personal como única medida de protección y los tiempos acelerados de producción son elementos que coadyuvan a aumentar las posibilidades de que los trabajadores se accidenten. Sobre todo se señaló que los ritmos acelerados de trabajo obstaculizan la posibilidad de que los trabajadores se protejan por sus propios medios. De esta manera, el tiempo de trabajo pasa a ser otro elemento que interviene en los procesos peligrosos derivados de la manipulación de agroquímicos. Este tiempo es una de las variables sobre la cual los empresarios inciden como forma de reducir costos y aumentar la competitividad $\mathrm{y}$, consiguientemente, la ganancia, en un sector donde los insumos así como los precios de venta son fijados internacionalmente. En el marco de la competencia entre empresas propia del sistema capitalista, reducir el tiempo de trabajo socialmente necesario aparece como una de las estrategias a desplegar. ${ }^{20}$

Estas formas de imposición que subyacen al trabajo asalariado en el sector condicionan los modos de vida, los procesos de saludenfermedad y las posibilidades de reproducción y desarrollo pleno de los trabajadores. ${ }^{7}$ Se trata de la imposición de un modelo de producción que adquiere características particulares en la ciudad 
de Young, pero que replica las generalidades del agronegocio a nivel internacional. Es éste el que genera las condiciones de posibilidad para las exposiciones eventuales de vecinos o escuelas rurales así como las crónicas en el caso de trabajadores. No es algo natural ni dado, sino que es consecuencia de la forma de producir riquezas, organizar el trabajo y cultivar cereales y oleaginosas con alta dependencia tecnológica. Morina y Cacace $^{4}$ afirman que la expansión del capitalismo a nivel agrario implicó el incremento de la composición orgánica del capital. Esto significó que la incorporación de nuevas tecnologías sustituyó la utilización de fuerza de trabajo, reconfigurando así las relaciones sociales de producción en el agro. De esta forma, la imposición de este modelo de producción, donde el uso intensivo de agroquímicos es uno de los aspectos, es un fenómeno históricamente situado y funcional a cierto desarrollo económico.

Breilh $^{7}$ afirma que los procesos que se presentan como permanentes o inherentes (lo que previamente fue caracterizado como imposición) requieren medidas profundas para ser modificados, por lo que no se solucionan con equipos de protección individual ni con cursos de capacitación para trabajadores. Esta perspectiva sólo responsabiliza a los asalariados por los efectos del modelo nocivo de producción. Por el contrario, es necesario analizar los elementos territoriales, sociales y económicos que generaron el aumento del uso de agroquímicos y su consiguiente contacto con la población trabajadora, para poder elaborar perfiles de intoxicación colectivos que permitan diseñar estrategias de prevención y promoción de la salud. La utilización de EPP, que fue señalada como proceso protector durante la investigación, está reglamentada como último recurso en la legislación uruguaya (Decreto 291/07). Si bien la legislación ofrece garantías a los trabajadores y se acerca a la línea de la prevención primordial o profunda propuesta por Breilh ${ }^{7}$, los establecimientos agrícolas relevados los utilizan como primer y único recurso. Redefinir las estrategias de prevención y promoción de la salud en el sector resulta imperioso cuando en la mayoría de los casos se desconocen los efectos crónicos de las sustancias químicas utilizadas, y aun no existen organizaciones sindicales que puedan demandar condiciones mínimas de trabajo seguro.

\section{CONCLUSIONES}

Del proceso de investigación se puede concluir que fue posible identificar perfiles de intoxicación colectivos que, como fue analizado, están determinados por el uso intensivo de agroquímicos que el modelo productivo promueve. Dichos perfiles permiten identificar patrones individuales de exposición. ${ }^{7}$ En el caso de aguateros, tractoristas y apoyos de tierra, trabajadores menos calificados del sector, la exposición es constante y la más acentuada en los cultivos de secano. De esta manera, la forma en la que se produce cereales con alta dependencia tecnológica, donde la imposición de los agroquímicos es uno de los aspectos, condiciona procesos destructores que atentan contra la salud de los trabajadores. Los niveles de riesgo tolerable, umbrales de seguridad, factores de riesgo o la eventualidad de los posibles daños son eufemismos que desdibujan la realidad de las condiciones de salud y de reproducción social de los asalariados del sector. La organización del trabajo actúa además como coadyuvante de los procesos destructores para la salud presentes en el proceso laboral.

La herramienta utilizada para la reconstrucción del proceso laboral y la identificación de procesos protectores y destructores permitió incorporar el saber de los directamente involucrados, y problematizar las formas de organizar el trabajo en el modelo productivo predominante. La instrumentación de espacios colectivos, además de producir conocimiento novedoso, promovió la sensibilización en torno a una problemática invisibilizada en el mundo del trabajo y a nivel social. En los estudios sobre salud y trabajo se está observando cierta tendencia a relegar los dispositivos grupales ante las dificultades que existen para su convocatoria y permanencia. En cambio, la tarea científica radica en ajustar los métodos de investigación-acción a los nuevos escenarios históricos, ya que la potencia transformadora de este tipo de herramientas de investigación colectiva no varía de acuerdo a los cambios en el entramado social.

Finalmente, seis años de investigar las condiciones de vida y de trabajo en asalariados del arroz, citrícolas y de cultivos de secano, han llevado a problematizar dos aspectos de la producción de conocimiento: por un lado, con quiénes investigamos y, por otro, qué sucede con el saber producido. El primer aspecto fue motivado por las debilidades en la organización sindical de los trabajadores rurales, y la imposibilidad de generar interlocutores constantes y a mediano plazo. A pesar de esto, la vulnerabilidad de estos trabajadores requiere del compromiso de otros actores sociales en la protección de derechos laborales: autoridades, académicos, otros trabajadores organizados, etc. Incluir a estos actores en los proyectos de investigaciónacción aparece como un elemento estratégico para alcanzar cambios y/o ajustes de los procesos nocivos de trabajo. Asimismo, una vez producido el conocimiento, el acompañar su instrumentación pública representa otro reto de las investigaciones en salud y trabajo, para así evitar una actitud contemplativa del fenómeno y poder avanzar en su transformación efectiva.

Se presentan parte de los resultados de la investigación "Salud laboral y ambiental en torno a la utilización de agroquímicos en la localidad de Young. Estrategias de monitoreo participativo", la cual fue aprobada y financiada por la Comisión Sectorial de Investigación Científica de la Universidad de la República en su convocatoria a Proyectos Orientados a la Inclusión Social, edición 2012. 
1. Oyhantçabal G, Narbando I. El Agronegocio y la Expansión del Capitalismo en el Campo Uruguayo. Rebela [en línea]. 2013; 2(3):409-425 [consultado jun 2016]. Disponible en: http://rebela.emnuvens.com.br/pc/article/viewFile/114/231.

2. Uruguay. Instituto Nacional de Estadistica. 2011. Censo 2011: microdatos [en línea] Montevideo: INE; 2011 [consultado may 2016]. Disponible en: http:// www5.ine.gub.uy/censos2011/resultadosfinales/rio\%20negro.html.

3. Abbate $S$, Colazzo $M$, Fonsalia $A$, Heinzen $H$, Heinzen $J$, Niell $S$, et al. Agroquímicos, salud laboral y ambiental: Diálogo de saberes y búsqueda de alternativas en una comunidad urbana del litoral del pais. Montevideo: Comisión Sectorial de Investigación Científica-Universidad de la República. De próxima aparición 2015.

4. Morina J, Cacace G. Capitalismo agrario y expansión sojera en la Argentina. Meridiano: Rev Geogr. 2013; 2:105-126.

5. Laurell AC, Noriega M. La salud en la fábrica. México, DF: ERA; 1989.

6. Betancourt 0. Enfoque alternativo de la salud y seguridad en el trabajo. Prevención es Desarrollo [en línea] 2009;1(1):3-16 [consultado jun 2016]. Disponible en: http://www.dso.fmed.edu.uy/sites/www.dso1.fmed.edu.uy/files/ materiales/Enfoque $\% 20$ alternativo\%20de\%20la\%20salud\%20y\%20seguridad\%20en\%20el\%20trabajo\%20-\%20Art\%C3\%ADculo_dr._Oscar_ Betancourt.pdf.

7. Breilh J. Epidemiología critica: Ciencia emancipadora e interculturalidad. Buenos Aires: Lugar Editorial; 2003.

8. Peres F, de Lucca SR, Ponte LM, Rodrigues KM, Rozemberg B. Percepção das condições de trabalho em uma tradicional comunidade agrícola em Boa Esperança, Nova Friburgo, Rio de Janeiro, Brasil. Cad Saúde Pública. 2004. 20(4):1059-1068.

9. Fonseca $M$, Peres $F$, Firmo J, Uchôa E. Percepção de risco: maneiras de pensar e agir no manejo de agrotóxicos. Cienc Saúde Colectiva. 2007;12(1):39-50.

10. Gasparini FM, De Freitas MC. Trabalho rural, saúde e ambiente: as narrativas dos produtores de flor frente aos riscos socioambientais. Ambient Soc. 2013; 16(3):23-44.

11. Rigotto R. Agrotóxicos, trabalho e saúde: vulnerabilidade e resistencia no contexto da modernizaçao agrícola no Baixo Jaguaribe/CE. Fortaleza: Ediçoes UFC; 2011.
12. Souza MC. La artesanía de la investigación cualitativa. Buenos Aires: Lugar Editorial; 2013.

13. Martínez S. La investigación participativa como práctica social y su aportación al mundo laboral a través del Modelo Obrero. Salud de los Trabajadores [en línea] 2007;15(2):107-118 [consultado jun 2016]. Disponible en: http://www.scielo.org. ve/scielo.php?script=sci_arttext\&pid=\$1315-01382007000200005\&lng=es\&n $\mathrm{rm}=$ iso\&tlng=es.

14. Alegre M, Fonsalía A, Frank N, Guigou B, Hahn M, Heinzen J et al. Abordaje de la salud laboral en los trabajadores del arroz desde una perspectiva interdisciplinaria: El Modelo Obrero como herramienta para la co-producción de conocimientos. Revista Digital Universitaria [en línea] 2012;13(5):16 [consultado may 2016]. Disponible en: http://www.revista.unam.mx/vol.13/num5/art54/index. html.

15. Guber R. El salvaje metropolitano: Reconstrucción del conocimiento social en el trabajo de campo. Buenos Aires: Paidós; 2004.

16. Hernández $R$, Fernández $C$, Baptista $P$. Metodología de la investigación. México: McGraw-Hill Interamericana; 2006.

17. Fals Borda, O. Origenes universales y los retos actuales de la IAP. Anal Polit [en línea] 1999; 38:71-88 [consultado may 2016]. Disponible en: http://datateca. unad.edu.co/contenidos/301500/301500_2015_Periodo_//Curso_Intervencion_ psicosocial_en_la_comunidad_Periodo_I_2015_/UNIDAD_II/Or_genes_ universales_y_retos_actuales_de_la_IAP.pdf.

18. Carneiro FF, Rigotto RM, Da Silva LG, Friedrich K, Búrigo AC. Dossie ABRASCO: Um Alerta sobre os impactos dos agrotóxicos na saúde [on-line]. São Paulo: Expressão Popular; 2015 [consultado may 2016]. Disponible en: http://abrasco. org.br/dossieagrotoxicos/.

19. Piñeiro D. Precariedad objetiva y subjetiva en el trabajo rural: nuevas evidencias. Rev Cienc Soc (Montevideo) [en línea]. 2011;24(28):11-33 [consultado jun 2016]. Disponible en: http://cienciassociales.edu.uy/wp-content/uploads/sites/3/2013/ archivos/RevCienSoc\%2028-2.pdf.

20. Alegre $M$, Fonsalia A, Frank N, Guigou B, Hahn M, Heinzen J, et al. Los trabajadores arroceros de la cuenca de la Laguna Merin: Análisis de su situación de salud. Montevideo: Espacio Interdisciplinario-Universidad de la República. De próxima aparición 2014. 